\title{
Epilepsy: A Stigma More than Disease
}

\section{Shoukat M and Iqbal RK* \\ Institute of Molecular Biology and Biotechnology, Bahauddin Zakariya University, Pakistan}

*Corresponding author: Rana Khalid Iqbal, Institute of Molecular Biology and Biotechnology, Bahauddin Zakariya University, Multan-60800, Pakistan, Tel: +92-61-9210463; Email: khalid.iqbal@bzu.edu.pk

\section{Abstract}

Epilepsy is a common neurological disorder that occurs from ancient times and accompanying with convulsions or seizures. Epilepsy has revealed a genetic basis. Epilepsy which is considered as a neurodevelopmental disorder has reduced the life expectancy and associated with various stigmatized attitudes or beliefs. Epilepsy and seizures can develop in any person both in male and female at any age. Head trauma and brain strokes are the major causes of epilepsy in adults. Epilepsy accompanied by changes in behavior, personality, and cognition. Several aspects of epilepsy can affect the brain and behavior. Stigma is a reality for a lot of people with a mental disorder. It is a mark of disgrace which sets a person apart from others. Negative attitudes and beliefs create prejudice which leads to negative actions and discrimination. Stigma and social exclusions are stereotyped characteristics of epilepsy. Someone with a mental illness known to be a dangerous and senseless rather than saying in poor health conditions. There are no effective cures for an epileptic people. Besides, many epileptic therapies or cures are still available for the diagnosis and prevention of people with epilepsy. Epilepsy treatment entails how epilepsy is treated and which techniques and antiepileptic drugs are used.

Keywords: Seizures; Epilepsy Stigma; Anti-Epileptic Drugs; Neurological Disorder; Epileptic People

\section{Introduction}

Epilepsy is a primitive disorder that has been described from ancient times to the modern day. Although, epilepsy is described by seizures which are incalculable in their occurrence and epilepsy is a stereotyped neurological disorder by which people of all ages are affected. Epilepsy takes in to account 22 million in the United States and $>65$ million people globally [1]. Besides the challenges associated with seizures and possessing chronic health disorders, epileptic people often encounter stigmatizing reactions and pessimistic stereotypes among the general public [2]. It is unfortunate that epilepsy is analogous with a reduction in life expectancy. People with epilepsy have neurodevelopmental disorders, usually concurrent with autism and physical impairment which shorten the life span [3]. Consequently, epilepsy inflicts a large economic burden on global health care systems and in low or middle-income countries (LMICs) it is a crucial public health problem [4]. Epilepsies may be upshot from primary and secondary genetic abnormalities to unambiguous structural and metabolic disorders, of which, some also possess genetic causes. It is considered that more than half of epilepsies hold a genetic basis. Eighty-four genes have been known as an epileptic gene. In total, 73 genes are categorized as neurodevelopmental associated epilepsy genes [5]. 


\section{Epilepsy Stigma}

In the particular part of the world and based on the culture of a specific era a lot of misconceptions about the condition were carried. These concepts and preconceived opinion that is not based on reason may lead to rejection, refusal of education and isolation mainly but not exclusively in the developing world. There is a conventional agreement that stigma and exclusion are stereotyped characteristics of epilepsy and a prime contributor to the burden related to the condition in both the developed and developing countries. Stigma promotes the hidden burden of illness which is a distinctive attribute of several chronic health problems. The UK Institute of Psychiatry Initiative, Mental Health Care, reveal that stigma can be perfectly defined under three words the ignorance, discrimination, and prejudice. Epilepsy has been debated horrified as well as a characteristic of the devil. Unless in the twenty-first century, in African Cameroon, people yet accept that an epileptic people are inhabited by devil; in Liberia the happening of epilepsy is associated with black magic and evil spirits and in Swaziland all traditional healers indicate the use of magic, especially black magic as the cause of epilepsy [6]. In the developing world the pressure of epilepsy influence the people with epilepsy (PWE), their family and their society in general. Epileptic people may be stigmatized and have a powerless position in society which is a physiological effect on people with epilepsy. Many particular aspects of life are influenced by epilepsy. Welfare security is endangered by epilepsy. Baskind and Birbeck have narrated three forms of stigmatization related to epilepsy; enacted, felt and courtesy. Enacted stigma refers to the experience of unfair treatment by others. Felt stigma occurs due to a fear of being discriminated against. Courtesy stigma is the public disapproval evoked as a result of associating with a stigmatized individual or a group when someone close to PWE (people with epilepsy) [7].

\section{Treatment or Medical Care for Epilepsy}

Sudden Unexpected Death in Epilepsy (SUDEP) is the main cause of death in patients who are suffered from persistent epilepsy, in other words, ungovernable epilepsy; approximately cause 10 to $50 \%$ deaths. There is no efficacious treatment or cure against SUDEP [3].

\section{Drug Therapy}

Usually, the cause of epilepsy is unresolved, in many cases as the area of the cerebral cortex that is essential for the generation of clinical seizures (epileptogenic focus) cannot be eliminated, antiepileptic drugs (AEDs) are used to govern seizures. Drugs against epilepsy are very beneficial in controlling seizures: $75 \%$ of people treated with antiepileptic drugs become seizure free; $20 \%-30 \%$ of PWE have impulsive, a period of temporary recovery from seizures without medication. Antiepileptic drug (AED) which is used extensively and low cost is Phenobarbital. It can manage a variety of seizures and it is on the significant drug list of $95 \%$ which is considered by the World Health Organization (WHO); Carbamazepine, Phenytoin and Valproate drug list in 93\%, 86\% and 87\% of countries, respectively or independently [4].

\section{Gene Therapy}

Antiepileptic drugs not at all can stop the occurrence of epilepsy in cases in which the source of the epileptogenic lesion is conspicuous (head trauma, stroke, episode of status epilepticus (SE), and brain infection). At the minimum $30 \%$ of, the epilepsies are considered as congenital. The single mutant gene is the basis of only infrequent forms of epilepsy, while more frequently; they are caused by the inheritance of two or more susceptibility genes. The single and first logical target for those individuals who are pharmacoresistant to epileptic seizures is the modulation of excessive sensitivity of an organ or a body part by enhancing the robustness of inhibitory signals or minimizing the strength of an excitatory signal. Efforts are being made to exploiting strategies for the administration of genes to the brain by crossing the blood-brain barrier (BBB) after the vector's administration in the peripheral blood for the treatment of epileptic seizures [8].

\section{Cognitive Therapy}

Cognitive behaviorism maintains those feelings or convictions that affect behavior and physiology. Cognitive behavioral therapy (CBT) or simply cognitive therapy explores the relationships between thoughts, seizure, and emotions which is frequently used in association with mind-body approaches. The purpose of cognitive therapy is to encourage the development of an increased sense of self-possession over the happening of the seizure [9].

\section{Electrical Stimulation of the Brain}

People who possess a variety of neurological disorders, neurostimulation represent a progressively interesting treatment. By the direct target on a particular neural region, neurostimulation can regulate a physical or cerebral feature which is regarded as an indicating a condition of disease in a way that is able to be adjusted, and changeable with the abolition of the implanted hardware. Furthermore, neurostimulation restraint many 
harmful effects that are commonly allied with medications. Treatment may be in the form of peripheral nerve stimulation such as spinal cord stimulation; vagus nerve stimulation (VNS); deep brain stimulation (DBS); or transcutaneous brain stimulation. The hippocampi and the adjoining nerves in the brain are the most seizure susceptible structures and these areas contain the substrate for the complex partial seizures which affects brain cells. In the original human neurostimulation studies, anterior nucleus of the thalamus (ANT) was one of the targets. Through the cortical stimulation drug, resistant motor seizures have been treated in six patients [10]. Computed tomography (CT) and magnetic resonance imaging (MRI) scans are important as a supplementary rather than essential to a therapeutic examination and EEG (Electroencephalogram) for the assessment of an individual with seizures [11].

\section{Conclusion}

In specific parts of world people yet accept that epilepsy is concerned with untrue myths like black magic, demons, and evil spirits. On the basis of present study findings, it can be concluded that epilepsy is not any kind of stigma which comes in many forms. It is just a misconception about epilepsy. Hence epilepsy is a neurodegenerative disorder that occurs as a result of seizures. Epilepsy can be better understood by having correct knowledge about this common neurological disorder. All possible treatments are available to cure a disorder of epilepsy.

\section{References}

1. Sirven JI (2015) Epilepsy: A Spectrum Disorder. Cold Spring Harb Perspect Med 5(9): a022848.
2. Sajatovic M, Herrmann LK, Doren JRV, Welter E, Perzynski AT, et al. (2018) HHS Public Access, 58(11), 1946-1954.

3. Jones LA, Thomas RH (2017) Sudden death in epilepsy : Insights from the last 25 years. Seizure 44: 232-236.

4. Mbuba CK, Newton CR (2009) Packages of Care for Epilepsy in Low- and Middle-Income Countries. PLoS Med 6(10): e1000162.

5. Wang J, Lin Z, Liu L, Xu H, Shi Y, et al. (2017) Epilepsyassociated genes. Seizure: European J Epilepsy 44: 11-20.

6. Boer HMD (2010) Epilepsy stigma: Moving from a global problem to global solutions. Seizure: European J Epilepsy 19(10): 630-636.

7. Boling W, Means M, Fletcher A (2018) Quality of Life and Stigma in Epilepsy, Perspectives from Selected Regions of Asia and Sub-Saharan Africa. Brain Sci 8(4): 59.

8. Simonato M (2014) Epilepsy \& Behavior Gene therapy for epilepsy. Epilepsy Behav 38: 125-130.

9. Leeman-markowski BA, Schachter SC (2018) Cognitive and Behavioral Interventions in Epilepsy. Current Neurol Neurosci Reports 17(42): 1-19.

10. Fisher RS, Velasco AL (2014) Electrical brain stimulation for epilepsy. Nature Reviews 10(5): 261270 .

11. Stafstrom CE, Carmant L (2015) Seizures and Epilepsy: An Overview for Neuroscientists. Cold Spring Harb Perspect Med 5(6): a022426. 